\title{
Cooperative space-time codes with network coding
}

\author{
Gordhan Das Menghwar ${ }^{*}$, Akhtar Ali Jalbani ${ }^{1}$, Mukhtiar Memon ${ }^{1}$, Mansoor Hyder ${ }^{1}$ \\ and Christoph F Mecklenbräuker ${ }^{2}$
}

\begin{abstract}
Because of the gains like diversity, throughput, and the coverage extension provided by the multiple-input multipleout (MIMO) systems, it has been part of the various wireless communications standards. However, to reap the benefits offered by MIMO systems in size-constrained nodes, used in wireless communications, has been a challenging issue. To address this challenge, cooperative communications has been introduced as an implementable way to exploit the multi-antenna (MIMO) gains in small size nodes. Various schemes or protocols have been devised to address the way by which cooperative communications actually takes place. In this article, we propose and investigate a novel scheme which provides a way to combine the benefits of space-time codes and network coding for cooperative communications. In this scheme, the cooperating users using space-time codes are assisted by the availability of a fixed relay node proposed for the future wireless networks. We compare our scheme with cooperative communications using Alamouti scheme and non-cooperative communications. With analytical results, we prove that the proposed scheme offers diversity gain of order three while cooperative communications using Alamouti scheme offers two and non-cooperative communications offers diversity order of one only. Moreover, the proposed relay assisted scheme outperforms by $15 \%$ in terms of multiplexing gain, the cooperative communications scheme being compared.
\end{abstract}

\section{Introduction}

Cooperative communications has been a hot topic for research since its introduction by Sendonaris et al. [1-3] in 1998. The reason for the popularity of the idea is that it gives a way to exploit the multiple-input multiple-out (MIMO) benefits in size-constrained nodes, like used in Ad hoc, sensor or a cellular network. It is well known that the wireless channel, while giving us independence of movement, also introduces an unreliability in the message being transmitted [4]. This unreliability is due to the inherent characteristics of the wireless channel like scattering, reflection, refraction, and diffraction. To overcome this problem, MIMO was introduced to use the spatial dimension, another degree of freedom, available in the wireless channel. MIMO uses multiple transmit and multiple receive antennas for transmission of the message and introduces independent fading paths between each transmit and receive antenna of the MIMO systems. In this way,

\footnotetext{
*Correspondence: gdas@sau.edu.pk

${ }^{1}$ Information Technology Centre, Sindh Agriculture University, Tandojam, Pakistan

Full list of author information is available at the end of the article
}

the receiver is provided with multiple copies of the same message from various statistically independent paths; this introduces spatial diversity gain in the wireless channel.

As for as point to point communications is concerned, this technique of MIMO system worked and has been part of many standards like UMTS [5], WiMAX [6], WLAN [7], and LTE-Advanced [8]. But due to the space problem, same happened to be challenging in size-constrained nodes. Cooperative communications is a concept to implement MIMO in the situation where it is not feasible to install multiple antennas on the nodes due to their size and the cost incurred per node.

Cooperative communications is a communications strategy where transmitting users use each others' antennas to realize MIMO gains. In cooperative communications when the transmitters send their messages to the destination, the free users present in their surrounding also receive that message. The users after detecting those messages, forward some additional information on behalf of the transmitting users to the destination. In this way, the receiver is provided with multiple copies of the same

\section{量 Springer}

C 2012 Das Menghwar et al. licensee Springer. This is an Open Access article distributed under the terms of the Creative Commons Attribution License (http://creativecommons.org/licenses/by/2.0), which permits unrestricted use, distribution, and reproduction in any medium, provided the original work is properly cited. 
message transmitted and the spatial diversity gain is introduced in the channel $[2,3,9]$. On the other hand, in a relay channel [10], a dedicated node is present between the transmitter and the receiver which is used to forward the second copy of the message transmitted by the user to the destination.

The way by which a relay node in a relay channel and the partner user in cooperative communications forward information to the destination is known as protocol. Some of the basic relaying protocols introduced so far are Decode-and-Forward, Compress-and-Forward, and Amplify-and-Forward [11,12]. In Decode-and-Forward relaying protocol, the relay node decodes and then reencodes the message received from the transmitting user and forwards it to the destination. In Amplify-andForward protocol, the relay node amplifies and forwards the signal received from the transmitting user to the destination. Finally, in Compress-and-Forward protocol, the relay node forwards the compressed version of the message received from the transmitting user to the destination.

We propose a scheme which is based on Decode-andForward protocol. Here the transmitting users, also called the partner users, act as relay nodes for each other. In addition, these cooperating users also exploit the availability of a dedicated relay node to cope with various adverse effects of the wireless channel and achieve the MIMO gains. Information theoretic outage probability and diversity multiplexing tradeoff are used as performance measures and the proposed scheme is compared with Alamouti scheme based cooperative communications and non-cooperative communications. The results achieved show that the proposed scheme offers higher diversity gain than non-cooperative as well as cooperative communications using Alamouti scheme.

The rest of the article is structured as follows. In Section System model, we describe our system model. Section Mutual information and the outage probability deals with the mutual information and the resulting outage probability of the proposed scheme. On the basis of the outage probability results in Section Mutual information and the outage probability, the diversity-multiplexing tradeoff perspective of the proposed scheme is presented in Section Diversity-multiplexing tradeoff. Finally, in Section Conclusion we conclude our article.

\section{System model}

To better explain our scheme, we take a cellular scenario with two mobile users $u_{1}$ and $u_{2}$ transmitting to a common destination, that is base station in this case. These transmitting users are considered to use space-time coding based Alamouti scheme for cooperation. In addition to that, we also introduce a dedicated relay node in the surrounding of the transmitting users and the scenario is depicted in Figure 1. The dedicated relay node is used to send network coded bits of the information received from the cooperating users to the destination. The network coded bits are formed by taking Exclusive-OR of the information bits received from the transmitting users. ${ }^{\text {a }}$

For better understanding, first we briefly discuss here the cooperative communications setup using Alamouti scheme only, this is depicted in Figure 2. The transmission of the message is accomplished in two phases. The first phase is the broadcast phase where mobile station users transmit their messages $s_{1}$ and $s_{2}$, respectively to the destination. Due to the broadcast nature of the wireless channel, these messages, in addition to the intended destination, are also received by each other and the scenario is illustrated in Figure 2a. The second phase is called the multiple-access phase. In this phase, the mobile station users, after detecting the messages from each other, send $-s_{2}^{*}$ and $s_{1}^{*}$, respectively to the destination. The messages sent in the multiple-access phase are formed by following well known space-time coding based Alamouti scheme [13]. The second phase of the transmission is shown in Figure $2 b$.

After having discussed this, now we explain our proposed scheme which we call as cooperative communications using Alamouti scheme with network coding. Again the whole process of communication spans two phases, i.e., the broadcast phase and the multiple access phase. This time in the first phase, the transmitted messages are not only received by the cooperating mobile station users and the intended destination but at a dedicated relay node,

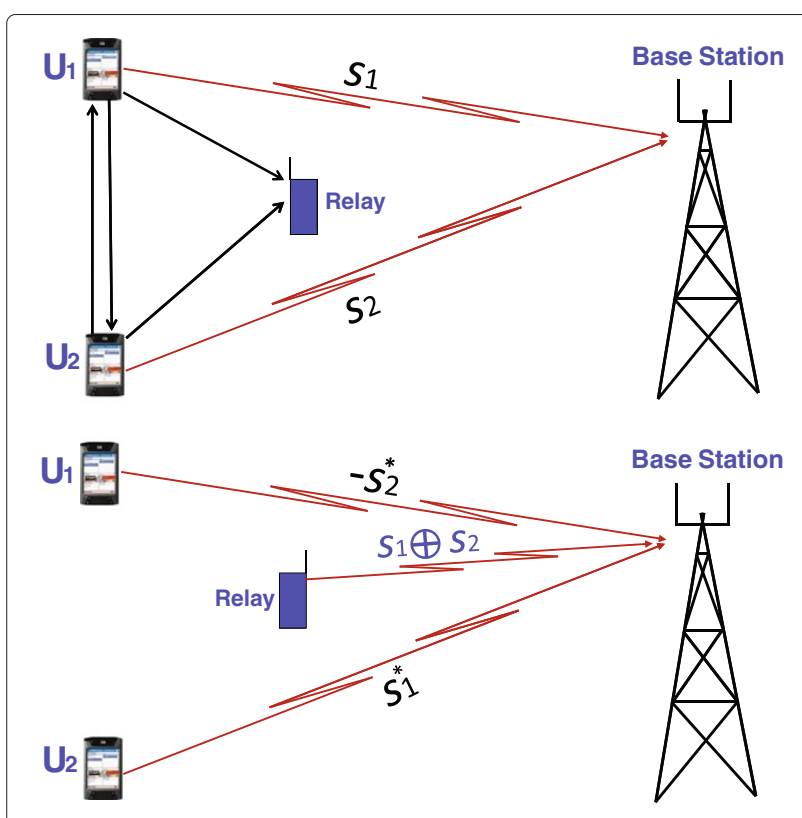

Figure 1 Alamouti scheme based cooperative communications with network coding. (a) Broadcast phase. (b) Multiple access phase. 

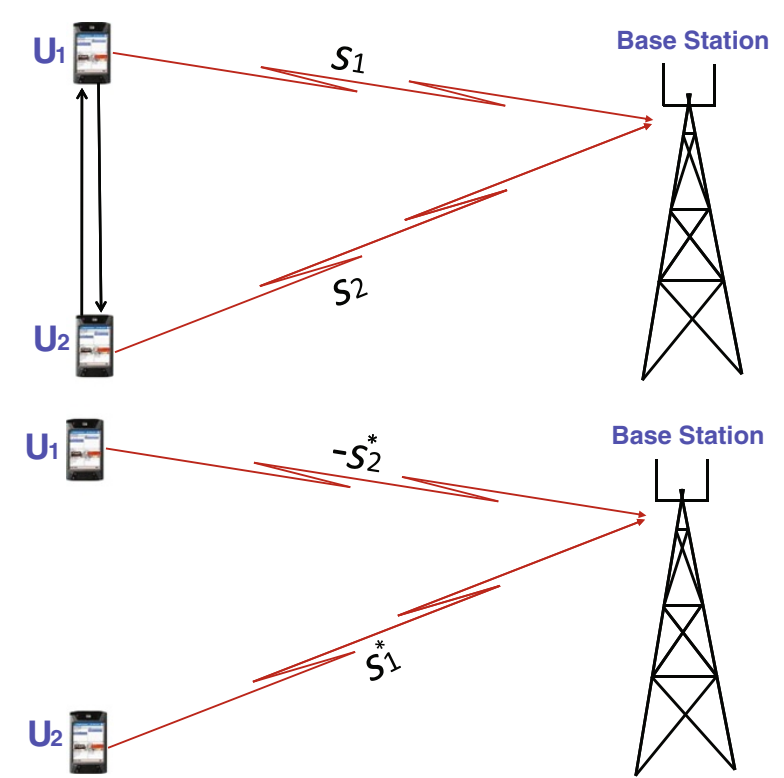

Figure 2 Alamouti scheme based cooperative communications.

present in the surrounding of the transmitting users, as well. This scenario is illustrated in Figure 1a. As shown, the transmitted messages are represented as $s_{1}$ and $s_{2}$.

In the next phase called the multiple access phase, the cooperating users $u_{1}$ and $u_{2}$, following Alamouti scheme, forward $-s_{2}^{*}$ and $s_{1}^{*}$, respectively to the destination. In addition to that in the multiple access mode, the available relay node sends the network coded bits of the information received from the cooperating users to the destination. This scenario is illustrated in Figure 1b.

In addition to the operational details of the scheme mentioned above, we assume that all the nodes operate in half duplex mode and all the transmitting terminal are assigned orthogonal channels by allocating separate time slots to the mobile station users and the relay node to transmit. The detailed description of the protocol showing the transmitting nodes, their channel assignments (time slots), and the respective transmitted messages are shown in Table 1.

In addition to that, every transmitting node is assumed to obey a fixed transmission power constraint of $P_{j}$ for

Table 1 Protocol description

\begin{tabular}{llll}
\hline Time slot & Transmitter & Message & Receiver \\
\hline 1 & $u_{1}$ & $s_{1}$ & $u_{2}, B S, r$ \\
2 & $u_{2}$ & $s_{2}$ & $u_{1}, B S, r$ \\
3 & $u_{1}$ & $-s_{2}^{*}$ & $B S$ \\
4 & $u_{2}$ & $s_{1}^{*}$ & $B S$ \\
5 & $r$ & $s_{1} \oplus s_{2}$ & $B S$ \\
\hline
\end{tabular}

their messages to be transmitted. Signal to noise ratio is defined as

$$
\mathrm{SNR}=\Xi\left|h_{j, d}\right|^{2},
$$

where $\Xi=\frac{P_{j}}{\eta}$ and $j=1,2$ represents the transmitting mobile station index. $\eta$ shows the noise introduced at the receiving end which is assumed to follow complex Gaussian distribution with zero mean and unit variance.

\section{Mutual information and the outage probability}

As detailed in Section System model, we consider a blockfading channel model. In this case, the channel is assumed to change its state from on channel realization to another. Since the mutual information is dependent on the channel gain between the transmitter and the receiver, the mutual information by itself is a random variable and does not support a constant rate requirement of certain application and the system is said to be in outage.

This section deals with the outage analysis of our proposed scheme and to calculate outage probability, we follow the definition from $[14,15]$ which is given as

$$
P[\Gamma]=P[I<\Gamma]
$$

That is, the outage probability $P[\Gamma]$, defines an event when the information theoretic mutual information $I$, is less than the required spectral efficiency, $\Gamma$ of the system. Therefore, to calculate the outage probability, first we need to find out the mutual information of the proposed scheme, which in our case becomes. ${ }^{b}$

$$
I=\frac{2}{5} \log \left(1+\frac{\mathrm{SNR}}{2} \sum_{i=1}^{2}\left|h_{i, d}\right|^{2}\right)+\frac{2}{5} \log \left(1+\mathrm{SNR}\left|h_{r, d}\right|^{2}\right)
$$

where $h_{i, d}$ is used to represent the channel gain from mobile station $i$ to the destination $d$, and $h_{r, d}$ is the channel gain from the relay node to the destination $d$. For simplification, the channel between the mobile station users is assumed to be error free. Equation (3) is the mutual information between user $u_{i}$ and the destination and as seen, the given expression is composed of two terms. The first term is because of the Alamouti scheme [16], where the transmitting and the partner user transmit using Alamouti scheme, and the second term shows here because of the relay node forwarding network coded bits to the destination [17]. We have introduced a factor of $\frac{2}{5}$ in front of mutual information to count the fact that five time slots are used by two cooperating users for the transmission of their messages to the destination $[17,18]$. 
Now by putting the value of mutual information (3) into (2), the outage probability of the proposed scheme is found as follows.

$$
\begin{aligned}
& P[\Gamma]=P[I<\Gamma] \\
& =P\left[\frac{2}{5} \log \left(1+\frac{\mathrm{SNR}}{2} \sum_{j=1}^{2}\left|h_{j, d}\right|^{2}\right)\right. \\
& \left.+\frac{2}{5} \log \left(1+\mathrm{SNR}\left|h_{r, d}\right|^{2}\right)<\Gamma\right] \\
& =P\left[\frac{2}{5} \log \left(1+\frac{\mathrm{SNR}}{2} \sum_{j=1}^{2}\left|h_{j, d}\right|^{2}\right)\right. \\
& \left.\times\left(1+\mathrm{SNR}\left|h_{r, d}\right|^{2}\right)<\Gamma\right] \\
& =P\left[\left(\left|h_{r, d}\right|^{2}+\frac{1}{2} \sum_{j=1}^{2}\left|h_{j, d}\right|^{2}\right.\right. \\
& \left.\left.+\frac{\mathrm{SNR}}{2}\left|h_{r, d}\right|^{2} \sum_{j=1}^{2}\left|h_{j, d}\right|^{2}\right)<\frac{2^{\frac{5 \Gamma}{2}}-1}{\mathrm{SNR}}\right]
\end{aligned}
$$

Let $\left|h_{r, d}\right|^{2}=y, \sum_{j=1}^{2}\left|h_{j, d}\right|^{2}=z$, and $\frac{2^{\frac{5 \Gamma}{2}}-1}{\mathrm{SNR}}=\delta$.

Then

$$
\begin{aligned}
P[\Gamma] & =P\left[y+\frac{1}{2} z+\frac{1}{2} \mathrm{SNR} y z<\delta\right] \\
& =P\left[y\left(1+\frac{1}{2} \mathrm{SNR} z\right)<\delta-\frac{1}{2} z\right] \\
& =P\left[y<\frac{\delta-\frac{1}{2} z}{1+\frac{1}{2} \mathrm{SNR} z}\right] \\
& =\int_{0}^{2 \delta} P\left[y<\frac{\delta-\frac{1}{2} z}{1+\frac{1}{2} \mathrm{SNR} z} \mid z\right] P_{Z}(z) d z \\
& =\int_{0}^{2 \delta}\left[1-\exp \left(-\lambda \frac{\delta-\frac{1}{2} z}{1+\frac{1}{2} \mathrm{SNR} z}\right)\right] P_{Z}(z) d z
\end{aligned}
$$

The expression just before Equation (4) is because of the total probability theorem. That is [19]

$$
\begin{aligned}
F_{X}(x) & =P[X \leq x] \\
& =\sum_{i}^{n} P\left[X \leq x \mid B_{i}\right] P\left(B_{i}\right)
\end{aligned}
$$

$B_{i}$ represent the mutually exclusive events. Moreover, in the last line, $y$ has been replaced by its probability distribution which actually is the cumulative distribution function (cdf) of a single exponential random variable. As mentioned before, the variable $z$ is the sum of two exponential random variable and $P_{Z}(z)$ is the pdf for the sum of two exponential random variables. For completeness, a general form for $P_{Z}(z)$ is given by [19].

$$
P_{Z}(z)=\left\{\begin{array}{cc}
\frac{\lambda}{(m-1) !}(\lambda z)^{m-1} \exp (-\lambda z) & \text { for } z>0 \\
0 & \text { for } z \leq 0
\end{array}\right.
$$

where $\lambda>0$ represents the parameter of an exponential distribution. Moreover, Equation (5) is a special form of Gamma distribution when the shape parameter is taken to be an integer.

\section{Results and discussion}

Figure 3 displays various outage probability curves ${ }^{\mathrm{c}}$ obtained by using the outage probability expression (4) evaluated using different SNR values and the SNR itself is defined by Equation (1). Moreover, to achieve these curves, without loss of generality, $\Gamma$ is fixed at $1 \mathrm{~b} / \mathrm{s} / \mathrm{Hz}$ and $\lambda$ is taken to be one.

In Figure 3, the slopes of various communication schemes show the diversity order of that particular scheme [20]. To give a comparative overview, we also show in Figure 3, the outage probability curves for non-cooperative and cooperative communications using Alamouti sheme only. As seen, the slopes of

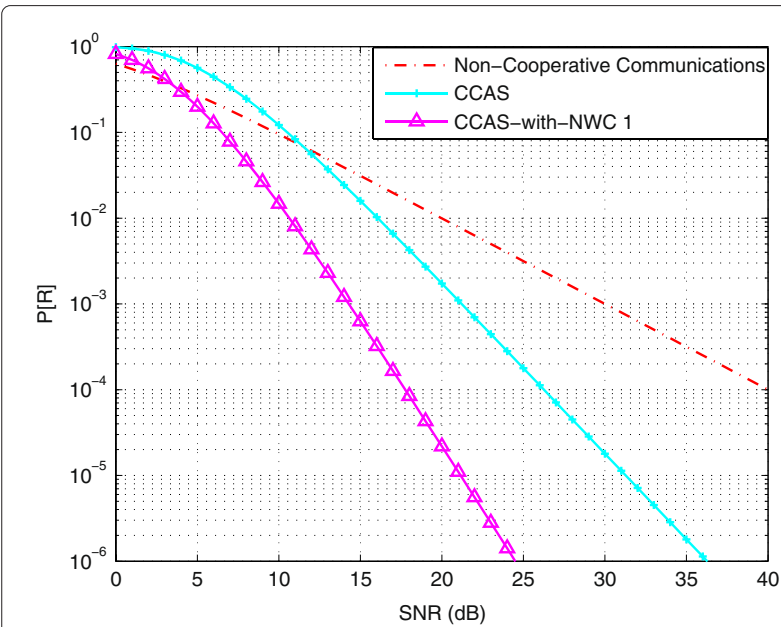

Figure 3 Outage probability for cooperative communications using Alamouti scheme with network coding when $\Gamma$ is fixed at $1 \mathrm{~b} / \mathrm{s} / \mathrm{Hz}$ and $\lambda$ is taken to be one. 
the outage probability curves indicate that the diversity order of non-cooperative and cooperative communications based on Alamouti scheme is one and two respectively. However, the diversity order of our proposed scheme, that is, cooperative communication using Alamouti scheme with network coding is three. This shows that the proposed scheme, in terms of the outage probability, outperforms both of the schemes being compared.

In addition to that, while most of the protocols do not offer gain in the low SNR regime, even they lose their performance in that region, the proposed scheme, as seen, offers better performance in that region as well. Normally low SNR term is used when the analytical expression are approximated in asymptotic sense. The remarkable work, in this direction, is done by Avestimehr and Tse [21]. However, in this article, by low SNR we imply the SNR in $d B$ where the cooperative communications using Alamouit scheme has low performance than non-cooperative communications. As seen in the figure, the proposed scheme offers no loss in this regime as well, where most of the protocols lose their performance. This happens between zero to $5 \mathrm{~dB}$, where the proposed scheme offers better performance than other cooperative communications scheme being compared, i.e, only Alamouti scheme based cooperative communications, as presented in Figure 2.

There is considerable work where people have tried to improve performance in the low SNR regime, but some of the notable work done in this direction is presented in $[22,23]$.

\section{Diversity-multiplexing tradeoff}

After having presented the outage behavior in the previous section, in this section we derive another performance measure called the diversity-multiplexing tradeoff perspective of our proposed scheme.

For this, we use the standard definition used by Zheng and Tse [24,25] and Menghwar [26] given as.

If a code family with a fixed block length for each SNR satisfies

$$
r=\lim _{\mathrm{SNR} \rightarrow \infty} \frac{R(\mathrm{SNR})}{\log (\mathrm{SNR})}
$$

then $r$ is known to be the multiplexing gain.

In the same way, if

$$
d=-\lim _{\mathrm{SNR} \rightarrow \infty} \frac{\log P_{e}(\mathrm{SNR})}{\log (\mathrm{SNR})}
$$

where $P_{e}$ is the probability of error, then $d$ is called the diversity gain.
Using the fact that the outage probability is the lower bound on the probability of error, Equation (7) is replaced by

$$
d=-\lim _{\mathrm{SNR} \rightarrow \infty} \frac{\log [P(I<\Gamma)]}{\log (\mathrm{SNR})} .
$$

The same approach has also been used in [14,27]. As seen, to use the above definition, we need the high SNR approximation of the outage probability. Therefore, we further simplify the outage probability results (4) of our scheme and get its high SNR approximation as follows.

$$
\begin{aligned}
P[\Gamma] & =\int_{0}^{2 \delta}\left[1-\exp \left(-\lambda \frac{\delta-\frac{1}{2} z}{1+\frac{1}{2} \mathrm{SNR} z}\right)\right] P_{Z}(z) d z \\
& =\int_{0}^{2 \delta}\left[1-\exp \left(-\lambda \frac{\delta-\frac{1}{2} z}{1+\frac{1}{2} \mathrm{SNR} z}\right)\right] \lambda \lambda z \exp (-\lambda z) d z \\
& \leq \int_{0}^{2 \delta}\left[\lambda \frac{\delta-\frac{1}{2} z}{1+\frac{1}{2} \mathrm{SNR} z}\right] \lambda^{2} z d z
\end{aligned}
$$

In the above simplification, the second equality of the expression (9) is obtained by using the actual form of $P_{Z}(z)$, i.e., the pdf for the sum of two exponential random variables. Finally, for the last inequality, the well known bounds $1-\exp (-z) \leq z, \forall z \geq 0$ and $\lambda \exp (-\lambda z) \leq \lambda$ have been used.

To proceed further, we use a mathematical technique called the change of variable is used to get the more simplified version of (9), specifically, putting $z=w \delta$ in (9) gives us.

$$
\begin{aligned}
P[\Gamma] & \leq \lambda^{2} \int_{0}^{1}\left[\frac{\delta-\delta w}{1+\mathrm{SNR} \delta w}\right] 2 \delta w 2 \delta d w \\
& =4 \lambda^{2} \delta^{3} \int_{0}^{1}\left[\frac{1-w}{1+t w}\right] w d w \\
& =4 \lambda^{2} \delta^{3} \int_{0}^{1}\left[\frac{1-w}{1+t w}\right] w d w \\
& =4 \lambda^{2}\left[\frac{2^{\frac{5}{2} \Gamma}-1}{\mathrm{SNR}}\right]^{3} \int_{0}^{1}\left[\frac{1-w}{1+t w}\right] w d w \\
& =4 \lambda^{2}\left[\frac{2^{\frac{5}{2} \Gamma}-1}{\mathrm{SNR}}\right]^{3} A(t)
\end{aligned}
$$

where

$$
A(t)=\int_{0}^{1}\left[\frac{1-w}{1+t w}\right] w d w \text { and } t=2^{\frac{5}{2} \Gamma}-1 .
$$


The above approximation (10) can further be simplified with the help of the bounds on $A(t)$ from [14] given as.

$$
\frac{1}{6+6 t} \leq A(t) \leq \frac{1}{2}
$$

Now by substituting bound (11) into (10) we get following asymptotic upper and lower bounds on (10) for outage probability which can be shown to be

$$
\begin{aligned}
& P[\Gamma] \geq\left[\frac{2^{\frac{5}{2} \Gamma}-1}{\mathrm{SNR}}\right]^{3} 2^{-\frac{5}{2} \Gamma} \frac{2 \lambda^{2}}{3}, \\
& P[\Gamma] \leq\left[\frac{2^{\frac{5}{2} \Gamma}-1}{\mathrm{SNR}}\right]^{3} 2 \lambda^{2} .
\end{aligned}
$$

Finally these bounds can readily be used in (8) to evaluate the required diversity-multiplexing tradeoff of the proposed scheme, i.e., cooperative communications using Alamouti scheme with network coding as follows.

\section{The upper bound on diversity-multiplexing tradeoff}

The achieved bounds (12) and (13) make it easy to use definition to (8) derive our second performance measure, i.e., diversity-multiplexing tradeoff of the proposed scheme. Specifically the lower bound (12) on the outage probability (4) will lead us to the upper bound on the diversity-multiplexing tradeoff as follows.

$$
\begin{aligned}
d & \leq-\lim _{\mathrm{SNR} \rightarrow \infty} \frac{\log \left[\left(\frac{2^{\frac{5}{2}} \Gamma-1}{\mathrm{SNR}}\right)^{3} 2^{\left.-\frac{5}{2} \Gamma \frac{2 \lambda^{2}}{3}\right]}\right.}{\log (\mathrm{SNR})} \\
& =-\lim _{\mathrm{SNR} \rightarrow \infty} \frac{\log \left(\frac{2^{\frac{5}{2}} \Gamma-1}{\mathrm{SNR}}\right)^{3}-\log \left(2^{\frac{5}{2} \Gamma}\right)+\log \left(\frac{2 \lambda^{2}}{3}\right)}{\log (\mathrm{SNR})} \\
& \leq-\lim _{\mathrm{SNR} \rightarrow \infty} \frac{3 \log \left(2^{\frac{5}{2} r \log \mathrm{SNR}}-1\right)-3 \log (\mathrm{SNR})-\log \left(2^{\frac{5}{2} r \log \mathrm{SNR}}\right)}{\log (\mathrm{SNR})} \\
& =-\lim _{\mathrm{SNR} \rightarrow \infty} \frac{3 \log \left(2^{\log S \mathrm{SNR}} \frac{5}{2} r^{5}-1\right)-3 \log (\mathrm{SNR})-\log \left(2^{\log S \mathrm{NR} \frac{5}{2}^{r}}\right)}{\log (\mathrm{SNR})} \\
& \leq-\left[\log \mathrm{SNR}(\mathrm{SNR})^{5 r-3}\right] \\
& =3-5 r
\end{aligned}
$$

The lower bound on diversity-multiplexing tradeoff In the same way, the upper bound (13) on the outage probability (4) is used to derive the lower bound on the diversity-multiplexing tradeoff which is given as

$$
\begin{aligned}
d & \geq-\lim _{\mathrm{SNR} \rightarrow \infty} \frac{\log \left[\left(\frac{2^{\frac{5}{2} \Gamma}-1}{\mathrm{SNR}}\right)^{3} 2 \lambda^{3}\right]}{\log (\mathrm{SNR})} \\
& =-\lim _{\mathrm{SNR} \rightarrow \infty} \frac{\left[3 \log \left(2^{2^{2} \Gamma}-1\right)-3 \log (\mathrm{SNR})+\log \left(2 \lambda^{3}\right)\right]}{\log (\mathrm{SNR})} \\
& \leq-\lim _{\mathrm{SNR} \rightarrow \infty} \frac{\left[3 \log \left(2^{\frac{5}{2} r \log \mathrm{SNR}}-1\right)-3 \log (\mathrm{SNR})\right]}{\log (\mathrm{SNR})} \\
& =-\lim _{\mathrm{SNR} \rightarrow \infty} \frac{\left[3 \log \left(2^{\log \mathrm{SNR}} \frac{5}{\frac{5}{2}^{2}}-1\right)-3 \log (\mathrm{SNR})\right]}{\log (\mathrm{SNR})} \\
& \geq-3\left[\log _{\mathrm{SNR}}(\mathrm{SNR})^{\frac{5}{2} r-1}\right] \\
& =3-\frac{15}{2} r .
\end{aligned}
$$

For simplification, from the second to the fifth line of expressions (14) and (15), we have used the basic log rules for multiplication and division. In addition to that, to arrive at the final expressions (14) and (15), we exploited the well known change of base formula.

\section{Results and discussion}

Figure 4 shows the diversity-multiplexing tradeoff curve for our proposed scheme along with the curves for cooperative communications using Alamouti scheme and noncooperative or direct transmission.

In the figure, on the horizontal axis, we show the multiplexing gain, $r$ and on the vertical axis, we show the diversity gain $d$.

As seen in Figure 4, both of the calculated upper and the lower bounds on the diversity-multiplexing tradeoff curves show that our proposed scheme offers the diversity gain of order three. In contrast, the maximum

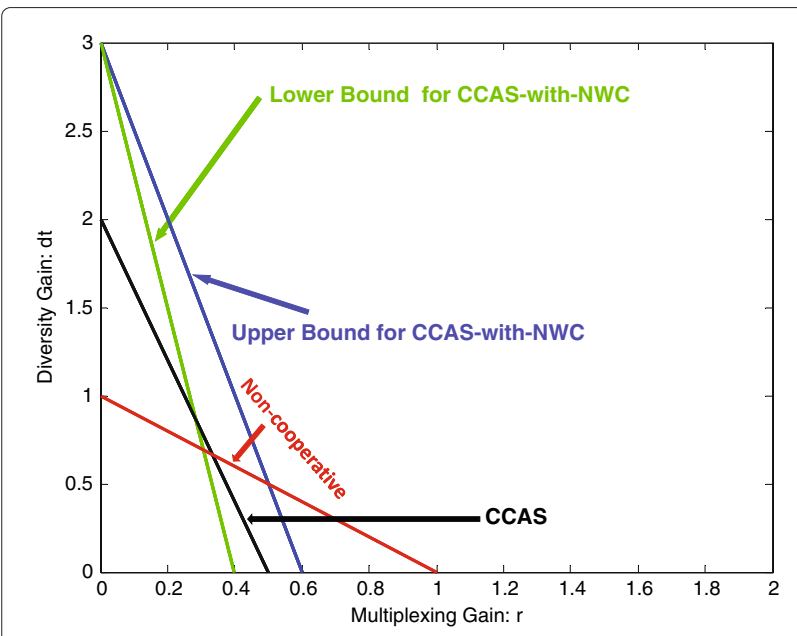

Figure 4 Diversity-multiplexing tradeoff for cooperative communications using Alamouti scheme with network coding. 
diversity gain achieved by Alamouti scheme based cooperative communications is two and with non-cooperative communications in one only.

Another perspective, that is of multiplexing gain $r$, shows that we get bounds, i.e., the upper and the lower bounds on the multiplexing gain. The Figure 4 shows that the upper bound on the multiplexing gain for our proposed scheme is $\frac{3}{5}$ and the lower bound is $\frac{2}{5}$, whereas, the maximum multiplexing gain for Alamouti scheme based cooperative communications is $\frac{1}{4}$ and for direct transmission is one. This shows that the proposed scheme output performs by $\% 15$ Alamouti scheme based cooperative communications.

\section{Conclusion}

In this article, we propose a novel scheme which exploits the availability of a relay node to aid a cooperative communications setup in a wireless network. To present the idea, we take two users Alamouti scheme based cooperative communication and a dedicated relay node in the network. With analytical results on the outage probability and the diversity-multiplexing tradeoff of the proposed scheme, we show that the proposed scheme outperforms Alamouti scheme based cooperative communications as well as non-cooperative or direct transmission. The results show that our proposed scheme offers diversity gain of order three in comparison with the diversity order of two and one offered by cooperative communications using Alamouti scheme and non-cooperative transmission respectively. The proposed scheme offers multiplexing gain of $\frac{3}{5}$ against $\frac{1}{4}$ offered by cooperative communications using Alamouti scheme only. In addition to that, while most of the existing schemes lose their performance in the low SNR regime, the proposed scheme offers no loss in the low SNR regime as well.

\section{Endnotes}

${ }^{a}$ In this article, the symbol of $\oplus$ is used to denote Exclusive-OR operationn.

${ }^{\mathrm{b}}$ In this article, all logarithms are taken to base 2, unless indicated explicitly.

${ }^{\mathrm{c}}$ In the figure, CCAS stands for cooperative communications using Alamouti scheme and CCAS-with-NWC stands for cooperative communications using Alamouti scheme with network coding.

\section{Competing interests}

The authors declare that they have no competing interests.

\section{Acknowledgements}

The authors would like to thank Prof. Dr. Gerald Matz, Institute of Telecommunications, Vienna University of Technology, Austria for his valuable discussions on information theoretic perspective of cooperative communications. They would also like to thank anonymous reviewers for their valuable comments to improve the technicality and readability of this manuscript.

\section{Author details}

${ }^{1}$ Information Technology Centre, Sindh Agriculture University, Tandojam, Pakistan. ${ }^{2}$ Institute of Telecommunications, Vienna University of Technology, Vienna, Austria.

Received: 3 October 2011 Accepted: 22 May 2012

Published: 2 July 2012

\section{References}

1. A Sendonaris, E Erkip, B Aazhang, in IEEE International Symposium on Information Theory. Increasing uplink capacity via user cooperation diversity (Cambridge, MA, 1998), p. 156

2. A Sendonaris, E Erkip, B Aazhang, User cooperation diversity-part I: system description. IEEE Trans. Commun. 51(11), 1927-1938 (2003)

3. A Sendonaris, E Erkip, B Aazhang, User cooperation diversity-part II: implementation aspects and performance analysis. IEEE Trans. Commun. 51(11), 1939-1948 (2003)

4. TS Rappaport (ed.), Wireless Communications: Principles and Practice (Prentice-Hall, Inc., Upper Saddle River, 1995)

5. 3GPP: Technical specification group radio access network; physical layer general description, Technical Report 25.201 V7.2.0, 3GPP, 2007

6. IEEE: IEEE standard for local and metropolitan area networks; part 16: air interface for fixed broadband wireless access systems, IEEE Std. 802.16-2004

7. IEEE: IEEE draft standard for local and metropolitan area networks; part 11: wireless LAN medium access control (MAC) and physical layer (PHY) specifications: amendment: enhancements for higher throughput, IEEE Draft Std.802.11n(d2) 2007

8. 3GPP TR 36814 V121: Further Advancements for EUTRA: Physical Layer Aspects. Technical Specification Group Radio Access Network 2009

9. GD Menghwar, CF Mecklenbräuker, Block-Markov encoding with network coding for cooperative communications. Elsevier J. Comput. Commun. 33, 2021-2030 (2010)

10. T Cover, AE Gamal, Capacity theorems for the relay channel. IEEE Trans. Inf. Theory. IT-25(5), 572-584 (1979)

11. JN Laneman, DNC Tse, GW Wornell, Cooperative diversity in wireless networks: efficient protocols and outage behavior. IEEE Trans. Inf. Theory. 50(12), 3062-3080 (2004)

12. G Kramer, M Gastpar, P Gupta, Cooperative strategies and capacity theorems for relay networks. IEEE Trans. Inf. Theory. 51(9), 3037-3063 (2005)

13. SM Alamouti, A simple transmit diversity technique for wireless communications. IEEE J. Sel. Areas Commun. 16(8), 1451-1458 (1998). [http://dx.doi.org/10.1109/49.730453]

14. JN Laneman, GW Wornel, Distributed space-time coded protocols for exploiting cooperative diversity in wireless networks. IEEE Trans. Inf. Theory. 49, 2415-2525 (2003)

15. GD Menghwar, CF Mecklenbräuker, in International ITG Workshop on Smart Antennas (WSA 2010). Outage analysis of cooperative space-time codes with network coding (Bremen, Germany, 2010), pp. 185-188

16. B Hassibi, BM Hochwald, High-rate codes that are linear in space and time. IEEE Trans. Inf. Theory. 48(7), 1804-1824 (2002)

17. X Bao, JL (Tiffany), Adaptive network coded cooperation (ANCC) for wireless relay networks: matching code-on-graph with network-on-graph. IEEE Trans. Wirel. Commun. 7(2), 574-583 (2008)

18. C Peng, Q Zhang, M Zhao, Y Yao, W Jia, On the performance analysis of network-coded cooperation in wireless networks. IEEE Trans. Wirel. Commun. 7(8), 3090-3097 (2008)

19. A Papoulis, Probability, Random Variables, and Stochastic Processes (Mc-Graw Hill, New York, 1984)

20. D Tse, P Viswanath, Fundamentals of Wireless Communication (Cambridge University Press, Cambridge, 2005)

21. AS Avestimehr, DNC Tse, Outage capacity of the fading relay channel in the low SNR regime. IEEE Trans. Inf. Theory. 53, 1401-1415 (2007)

22. C Hucher, GRB Othman, JC Belfiore, How to solve the problem of bad performance of cooperative protocols at low SNR. EURASIP J. Adv. Signal Process. 2008, 24 (2008)

23. C Hucher, GRB Othman, JC Belfiore, in IEEE International Symposium on Information Theory, 2007 (ISIT 2007). AF and DF protocols based on alamouti ST Code (Nice, France, 2007), pp. 1526-1530 
24. L Zheng, D Tse, in IEEE International Symposium on Information Theory (ISIT). Diversity and freedom: a fundamental tradeoff in multiple antenna channels (Lausanne, Switzerland, 2002), p. 476

25. L Zheng, DNC Tse, Diversity and multiplexing: a fundamental tradeoff in multiple-antenna channels. IEEE Trans. Inf. Theory. 49(5), 1073-1096 (2003)

26. GD Menghwar, in 17th European Wireless 2011, (EW-2011). Alamouti based cooperative communications witha relay node using network coding: a diversity-multiplexing tradeoff perspective (Vienna, Austria, 2011), pp. 1-4

27. GD Menghwar, CF Mecklenbräuker, in 3rd International Symposium on Applied Sciences in Biomedical and Communication Technologies (ISABEL 2010). Outage probability of alamouti based cooperative communications with multiple relay nodes using network coding (Rome, Italy, 2010), pp. 1-5

doi:10.1186/1687-1499-2012-205

Cite this article as: Das Menghwar et al:: Cooperative space-time codes with network coding. EURASIP Journal on Wireless Communications and Networking 2012 2012:205

\section{Submit your manuscript to a SpringerOpen ${ }^{\circ}$ journal and benefit from:}

- Convenient online submission

- Rigorous peer review

- Immediate publication on acceptance

- Open access: articles freely available online

- High visibility within the field

- Retaining the copyright to your article

Submit your next manuscript at $>$ springeropen.com 